\title{
Ramadan fasting among advanced chronic kidney disease patients
}

\author{
Nephrologists' perspectives in Saudi Arabia
}

Luluh Y. Alsughayer, MBBS, Lamees A. Altamimi, MBBS, Ahmad R. Tarakji, MD, FRCPC.

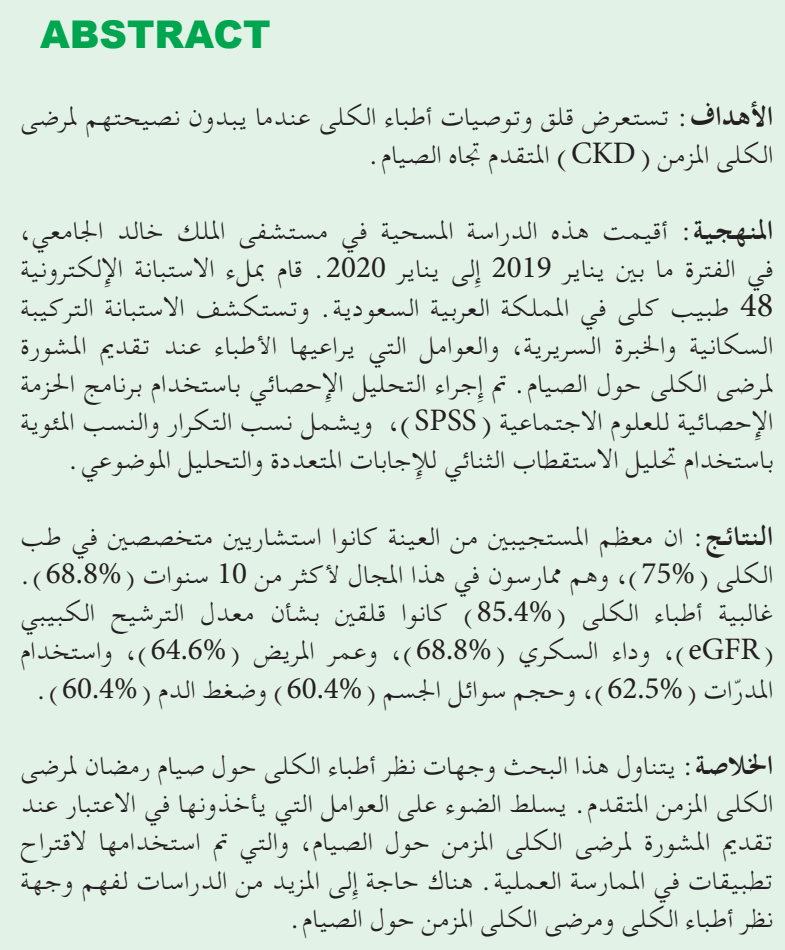

Objectives: To explore nephrologists' concerns and recommendations in counseling their advanced chronic kidney disease (CKD) patients on Ramadan fasting.

Methods: This cross-sectional study was carried out in King Khalid University Hospital, Riyadh, Saudi Arabia between January 2019 and January 2020. An electronic survey was completed by 48 nephrologists practicing in Saudi Arabia. The questionnaire focused on demographics, clinical experience, and factors to consider when advising CKD patients about fasting. Statistical analysis was performed by the Statistical Package for Social Sciences for Windows, version 21 (IBM Corp, Armonk, NY, USA). Statistics include frequency and percentages using multiple response dichotomy analysis and thematic analysis.
Results: Most respondents were consultant nephrologists (75\%), practicing nephrology for over 10 years $(68.8 \%)$. The majority of responding nephrologists $(85.4 \%)$ were concerned about estimated glomerular filtration rate (eGFR), diabetes $(68.8 \%)$, patient age $(64.6 \%)$, use of diuretics $(62.5 \%)$, body fluid volume $(60.4 \%)$, and blood pressure (60.4\%).

Conclusion: This research addresses nephrologists' perspectives about Ramadan fasting for patients with advanced CKD. It highlights factors they consider when advising CKD patients about fasting, which were used to suggest applications in practice. Further studies are needed to comprehend nephrologists' and CKD patients' perspectives on fasting.

Keywords: renal insufficiency, chronic, Islam, fasting, nephrologists, glomerular filtration rate

\section{Saudi Med J 2020; Vol. 41 (10): 1070-1075 doi: 10.15537/smj.2020.10.25378}

From the College of Medicine (Alsughayer, Altamimi), and from the Department of Medicine, College of Medicine (Tarakji), King Saud University, Riyadh, Kingdom of Saudi Arabia.

Received 28th June 2020. Accepted 1st September 2020.

Address correspondence and reprint request to: Dr. Ahmad R. Tarakji, Nephrology Unit, Department of Medicine, College of Medicine, King Saud University, Riyadh, Kingdom of Saudi Arabia. E-mail:atarakji@ksu.edu.sa

ORCID ID: https://orcid.org/0000-0001-8806-4911

Disclosure. This study was funded by the Deanship of Scientific Research at King Saud University Undergraduate Student's Research Support Program, Project No.: (URSP - 5 - 20 -01). 
$\mathrm{R}_{\mathrm{t} \text { tis }}$ amadan fasting represents one of the 5 pillars of the Islamic religion and is obligatory for healthy Muslims. Fasting is the practice of abstaining from food and drink from dawn to dusk. Ramadan is the ninth lunar month, that varies each year in timing. Based on geographical location and season, the duration of fasting ranges from 12 to 14 hours a day. ${ }^{1}$ Fasting is preceded by a meal at dawn (Sohoor) and ended with a meal at sunset (Iftar).

Chronic kidney disease $(\mathrm{CKD})$ has an estimated global prevalence of 11 to $13 \%$, with most patients having stage $3 \mathrm{CKD} .{ }^{2}$ Muslim CKD patients frequently ask their physicians regarding fasting; indeed, while not mandatory for sick people, many patients choose to fast. Muslims constitute over 23\% of the global population, yet there is a lack of evidence and protocols to guide practitioners' care of CKD patients during Ramadan. ${ }^{3,1}$ Therefore, different nephrologists have different approaches, and to our knowledge no previous study was published looking at nephrologists' point-of-view.

The effect of fasting on renal function for CKD patients is controversial. ${ }^{4}$ While some studies concluded that CKD patients can safely fast for Ramadan, others showed evidence of worsening renal function. ${ }^{5-7}$ Most studies were conducted during the cold season and were limited by small sample sizes and lack of control groups. However, in a meta-analysis, Bragazzi ${ }^{4}$ stated that no impact of seasonality was found by sensitivity analysis.Ultimately, the implementation of guidelines is needed to counsel fasting CKD patients and monitor them before, during, and after fasting..$^{1,4}$ Therefore, this research aims to explore factors influencing nephrologists' advice on fasting during Ramadan for patients with advanced CKD.

Methods. Literature search was performed through PubMed and Google Scholar. Relevant articles from 2015 to present were accessed using the keywords "fasting" and "chronic kidney disease". The references of retrieved articles were further reviewed for relevant publications.

This cross-sectional survey was carried out in King Khalid University Hospital (KKUH), Riyadh, Saudi Arabia, between January 2019 and January 2020. Data was collected via convenient sampling in 2 phases (Figure 1). In the first phase, nephrologists' contact information was provided by the Saudi Society of Nephrology and Transplantation. We included physicians practicing nephrology in Saudi Arabia, excluding pediatrics and pathology physicians who do not treat fasting CKD patients. Out of 68 practitioners contacted, 32 completed the questionnaire. For the second phase, the Saudi Center for Organ Transplantation provided a database of 104 additional nephrologists' emails, from which 16 responses were received. As such, this study's total sample was 48 participants, representing a response rate of $28 \%$.

The questionnaire was developed by the authors and sent to 2 nephrology consultants for revision and feedback. The questionnaire is composed of 3 sections, with 16 items in total. The first section asked respondents on the level of clinical experience, duration of nephrology clinical practice, age group, religion, and whether they had a special renal clinic to advise CKD patients regarding fasting for Ramadan. The second section listed factors that nephrologists may consider when advising CKD patients on fasting. The third section featured 2 patient case scenarios (Table 1); participants were asked if they would advise the patient

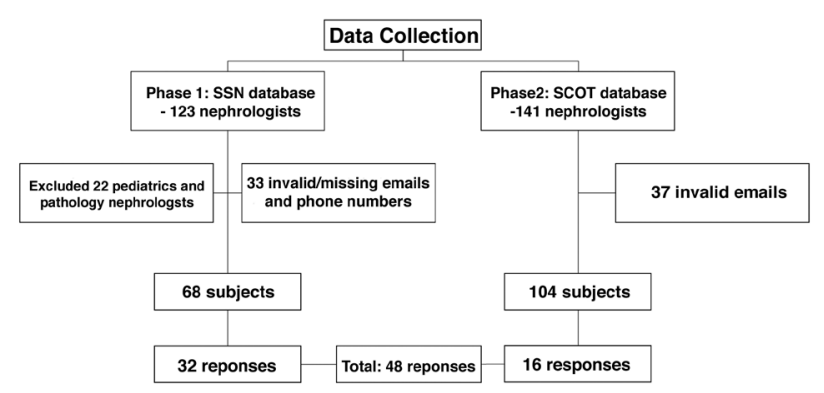

Figure 1 - Subjects recruitment and data collection process. SSN: Saudi Society of Nephrology, SCOT: Saudi Center for Organ Transplantation

Table 1 - Case scenarios used in the questionnaire.

Case scenario no. 1

The first case was a 50-year old Saudi female with stage 5A3 chornic kidney disease $(\mathrm{CKD})$, associated with complicated diabetes type 2 (retinopathy, neuropathy, nephropathy), hypertension, dyslipidemia, anemia, CKD-mineral and bone disorder, and morbid obesity (BMI 54 $\mathrm{kg} / \mathrm{m}^{2}$ ). She is taking Nifedipine XR, Aspirin, Atorvastatin, Carvedilol, Furosemide, Insulin, Darbepoetin, Calcium Resonium, Ferrous sulfate, and calcium carbonate. Her blood pressure is $137 / 73 \mathrm{mmHg}$ using large cuff. She has mild pitting edema. Her eGFR by CKD-Epi equation is $10.7 \mathrm{~mL} / \mathrm{min} / 1.73 \mathrm{~m}^{2}$ with serum creatinine of $448 \mathrm{umol} / \mathrm{L}$. Her serum creatinine has been slowly increasing over the last 3 years (299-384- 448 umol/L), though she denies any uremic symptoms. She is planning to fast during Ramadan as she did last year with no problems.

Case scenario no. 2

The second case was a 55-year-old male with stage 3 bA3 CKD due to membranous nephropathy. As he developed side effects to steroids, he instead takes Mycophenolate Mofetil with stable renal function $\left(\mathrm{eGFR}=43 \mathrm{~mL} / \mathrm{min} / 1.73 \mathrm{~m}^{2}\right.$, serum creatinine=142 umol/L). His proteinuria has decreased from $17 \mathrm{gr} /$ day to approximately $6 \mathrm{gr} / \mathrm{day}$. $\mathrm{He}$ is also on Atorvastatin, Irbesartan, Amlodipine, Cholecalciferol, and Levothyroxine. 
to fast and provide their reasoning via checklist, along with recommendations regarding diet, medication, lifestyle, and follow-up. An optional text box was left for further comments.

Participants did not receive any incentives or rewards. No personal data was collected from the subjects, and participants' privacy was assured. Ethical approval for this study was obtained from the Institutional Review Board of College of Medicine, King Saud University, Riyadh, Saudi Arabia.

Statistical analysis. The data was analyzed by using the Statistical Package for Social Sciences statistics for Windows, version 21 (IBM Corp, Armonk, NY, USA). Frequency and percentages were used to describe categorical factors. The multiple response dichotomy analysis was used to describe responses to multiple choice questions. The thematic analysis was used to categorize physicians' further narrative advice to fasting patients, and the multiple response dichotomy analysis was used to describe density (example, percentage) of these themes.

Results. Among the 48 nephrologists responding to the survey, most were either under 45 years old or over 54 years old (each 39.6\%), while the remainder $(20.8 \%)$ were 45 to 54 years old. All respondents were Muslim except one. Regarding clinical experience, most respondents were consultant nephrologists (75\%) and had practiced nephrology for over 10 years (68.8\%). Almost half of these respondents $(41.7 \%)$ have a dedicated renal clinic to discuss Ramadan fasting with CKD patients (Table 2).

Nephrologists were asked to select all medical factors they consider when advising CKD patients on Ramadan fasting (Figure 2). Most nephrologists were concerned regarding the patient's estimated glomerular filtration rate (eGFR) (85.4\%), presence of concomitant diabetes $(68.8 \%)$, patient age $(64.6 \%)$, diuretic use $(62.5 \%)$, body fluid (60.4\%), and blood pressure (60.4\%). Nephrologists also suggested they would consider the presence of comorbid heart failure (47.9\%), hyperkalemia (45.8\%), and patient adherence (45.8\%); less frequently, physicians were concerned on metabolic acidosis (39.2\%) and proteinuria (22.9\%).

Two medical case scenarios were then presented to the respondents (Table 1). For the first case, most nephrologists $(89.6 \%)$ advised the female patient not to fast due to risk of hypoglycemia $(70.8 \%)$, hyperkalemia (47.9\%), and hypervolemia (35.4\%). When asked to select advice had the patient insisted on fasting, respondents advised that she should limit potassium-
Table 2 - Nephrologists' professional and demographic characteristics $(\mathrm{N}=48)$.

\begin{tabular}{lc}
\hline Characteristics & $\mathbf{n}(\%)$ \\
\hline Age group & \\
$\quad<45$ years & $19(39.6)$ \\
$45-54$ years & $10(20.8)$ \\
$\quad>54$ years & $19(39.6)$ \\
Religion & \\
$\quad$ Non-Muslim & $1(2.1)$ \\
$\quad$ Muslim & $47(97.9)$ \\
Clinical Role & \\
$\quad$ Registrar nephrologist & $5(10.4)$ \\
Fellow and senior registrar nephrologist & $7(14.6)$ \\
$\quad$ Consultant nephrologist & $36(75.0)$ \\
Experience in nephrology (years) & \\
$\quad \leq 10$ years & $15(31.3)$ \\
$\quad>10$ years & $33(68.8)$ \\
Do you have nephrology clinic to advise patients & \\
on fasting? & \\
$\quad$ No & $28(58.3)$ \\
$\quad$ Yes & $20(41.7)$ \\
\hline
\end{tabular}

rich food $(83.3 \%)$, restrict salt-rich foods, and monitor body weight (79.2\%). In addition, $97.9 \%$ agreed that she should stop fasting if she developed signs of hypoglycemia and hypotension, such as dizziness. Most respondents $(91.7 \%)$ indicated that she should go to the emergency room (ER) if she developed uremic symptoms, such as nausea, vomiting, and shortness of breath. Also, most respondents (79.2\%) stated that lab tests should be thoroughly checked at the end of the first week of Ramadan (Table 3).

As for the second case, most nephrologists (83.3\%) agreed on allowing this male patient to fast; with concerns of hyperkalemia (18.8\%) and hypervolemia (16.7\%). The patient was advised to restrict potassium-rich food $(52.1 \%)$ and salt-rich food (81.3\%). Most nephrologists $(83.3 \%)$ advised him to break his fasting in emergency situations, such as dizziness. Only $16.7 \%$ of nephrologists advised him to decrease amlodipine dosage by half, but nearly all agreed he should adhere to Mycophenolate at Iftar and Sohoor (93.8\%). Over half $(58.3 \%)$ of nephrologists asked the patient to attend renal function test check-up after the first week of Ramadan (Table 3).

When asked for further advice in both cases, nephrologists' narrative recommendations for fasting CKD patients were: "take a leave from work and stay in cool environment"; "appropriate hydration during the night"; "avoid nephrotoxic agents"; "avoid high protein and salt intake"; "monitor body weight and edema of the lower limbs"; and "monitor blood pressure and glucose". ${ }^{8}$ 


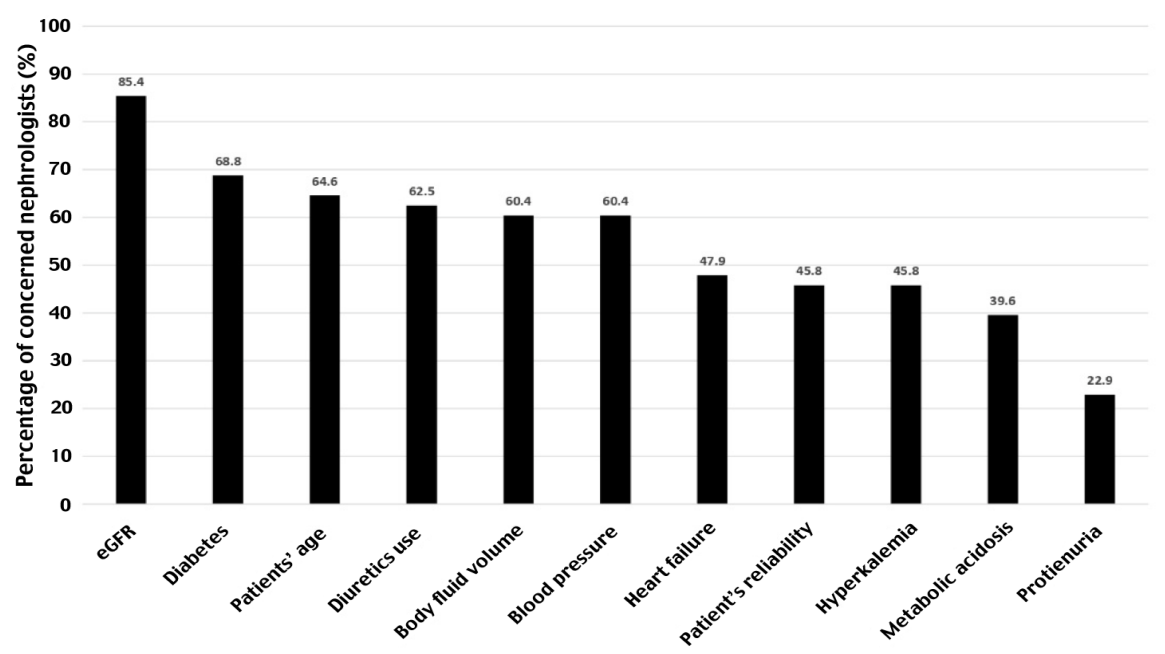

Concerning factors when advising CKD patients on fasting

Figure 2 - Factors that nephrologists consider when recommending fasting Ramadan for CKD patients. CKD: chronic kidney disease, $\mathrm{X}$ axis: concerning factors when advising $\mathrm{CKD}$ patients about fasting, $Y$ axis: percentage of concerned nephrologists (\%)

Discussion. The Islamic religion exempts chronically ill patients from fasting, especially those for whom fasting may pose negative health consequences. Religious scholars rely on advice from physicians in issuing decrees regarding exemption from Ramadan fasting. However, for CKD patients, the distinction between who can and cannot fast has been unclear.

Previous studies showed contradictory results regarding the impact of fasting on CKD patients and no approved guidelines exist. ${ }^{1}$ As such, nephrologists have different perspectives, and this study was aiming to explore Nephrologists' point-of-view regarding Ramadan fasting advice for patients with advanced CKD.

Our results showed that eGFR was the most important factor upon which nephrologists rely when assessing the appropriateness and impact of fasting. Most nephrologists advise stage $3 \mathrm{CKD}$ patients to fast, but not stage $5 \mathrm{CKD}$ patients. Indeed, the literature shows that more advanced CKD stage was independently associated with worsening renal function. ${ }^{7}$ The risk of developing acute kidney injury (AKI) was associated with a baseline creatinine clearance of less than $60 \mathrm{ml} / \mathrm{min} / 1.73 \mathrm{~m}^{2} .{ }^{9}$ Meanwhile, few studies found no significant GFR change between fasting and non-fasting CKD patients, or GFR improvement in fasting CKD patients. ${ }^{5,6,10,11}$

Comorbid diabetes was the second most important factor in discouraging fasting. According to the International Diabetes Federation and Diabetes and Ramadan International Alliance (IDF-DAR)
Table 3 - Case-based advice to patients with advanced CKD attempting to fast Ramadan

\begin{tabular}{|c|c|c|}
\hline Questionnaire items & $\begin{array}{c}\text { Case scenario } \\
\text { no. } 1\end{array}$ & $\begin{array}{l}\text { Case scenario } \\
\text { no. } 2\end{array}$ \\
\hline \multicolumn{3}{|c|}{ Would you advise the patient to fast Ramadan? } \\
\hline Yes & $5(10.4)$ & $40(83.3)$ \\
\hline \multicolumn{3}{|l|}{ Reasons why you advise avoiding fasting } \\
\hline Risk of Hyperkalemia & $23(47.9)$ & $9(18.8)$ \\
\hline Risk of Hypervolemia & $17(35.4)$ & $8(16.7)$ \\
\hline Risk of Hypoglycemia & $34(70.8)$ & NA \\
\hline \multicolumn{3}{|l|}{ Advice to the fasting CKD patient } \\
\hline Avoid fatigue and dehydration* & $16(76.2)$ & $9(60.0)$ \\
\hline $\begin{array}{l}\text { Restrict potassium-rich food such as } \\
\text { dates }\end{array}$ & $40(83.3)$ & $25(52.1)$ \\
\hline Restrict salt and watch your weight & $38(79.2)$ & $39(81.3)$ \\
\hline $\begin{array}{l}\text { Break your fast for emergency such as } \\
\text { dizziness }\end{array}$ & $47(97.9)$ & $40(83.3)$ \\
\hline $\begin{array}{l}\text { Go to emergency room if you develop } \\
\text { uremic symptoms }\end{array}$ & $44(91.7)$ & NA \\
\hline Proper diabetic management* & $18(85.7)$ & NA \\
\hline $\begin{array}{l}\text { Monitor fluid intake, output, and } \\
\text { blood pressure * }\end{array}$ & $8(38)$ & $10(66.7)$ \\
\hline Decrease amlodipine dose by half & NA & $8(16.7)$ \\
\hline $\begin{array}{l}\text { Take Mycophenolate at Iftar and } \\
\text { Sohoor }\end{array}$ & NA & $45(93.8)$ \\
\hline \multicolumn{3}{|l|}{ When do you want to check lab tests? } \\
\hline $\begin{array}{l}\text { At the end of the first week of } \\
\text { Ramadan }\end{array}$ & $38(79.2)$ & $28(58.3)$ \\
\hline In the middle of Ramadan & $13(27.1)$ & $22(45.8)$ \\
\hline At the end of Ramadan & $8(16.7)$ & $6(12.5)$ \\
\hline After Eid holiday & $8(16.7)$ & $13(27.1)$ \\
\hline
\end{tabular}

${ }^{*}$ Calculated by thematic analysis of textual further advice. Values are presented as numbers and percentage (\%) of nephrologists in favor of the statement. CKD: chronic kidney disease, NA: not applicable 
guidelines, diabetic patients with CKD who are willing to fast are classified as high-risk (CKD stage 3 ) and very high-risk (CKD stage 4/5). ${ }^{12}$ However, a prospective observational study of 68 diabetic CKD fasting patients found no significant difference in glycated hemoglobin or hypoglycemia between fasting and non-fasting groups. ${ }^{6}$

Patient age was another concern for nephrologists. Previous studies showed conflicting findings as worsening renal function was associated with younger age in one study and older age in another. ${ }^{7,5}$

Although blood pressure was a concern for our nephrologists, fasting was not shown by Chowdhury et $\mathrm{al}^{6}$ to adversely affect blood pressure. Fasting was also shown to lower systolic and diastolic blood pressures. ${ }^{11}$ However, higher baseline systolic blood pressure was associated with worsening renal function. ${ }^{7}$ Anti-hypertensive medications, namely diuretics, were associated with $\geq 25 \%$ drop in eGFR. ${ }^{5}$

While nephrologists were concerned on comorbid heart failure, in one study, fasting was not associated with an increase in cardiovascular events. ${ }^{6}$ Instead, fasting improved hydration status indices and plasma brain natriuretic peptide (BNP) levels in sufficiently hydrated CKD patients. ${ }^{10}$ However, the risk of significant hypovolemia remains, predisposing patients to worsening renal function. ${ }^{10}$ Recovery of renal function upon completion of fasting supports the role of dehydration. ${ }^{9}$

Patient adherence to diet, rest, hydration, and medications is critical. Culturally, there is a trend of consuming excessive amounts of carbohydrates and fatty foods at Iftar. This practice was discouraged by some of our study participants for CKD patients. Likewise, patients were advised to avoid vigorous exercise, especially in hot environments. Proper hydration during the night is also vital to avoid the risk of hypovolemia.

Chronic kidney disease patients are usually asked to avoid consumption of dates, which are high in potassium, to decrease the risk of hyperkalemia. However, fasting was found to have no impact on potassium, sodium, calcium, phosphorous, or uric acid levels in CKD patients. ${ }^{7}$ In addition, development of hyperkalemia was not associated with worsening renal function. ${ }^{7}$

Lastly, proteinuria was the least important consideration among our nephrologists. Ramadan fasting was associated with improvement of proteinuria in one study. ${ }^{5}$

Patients with advanced CKD should receive careful follow-up during their fasting month. The more complex the case, the earlier the patient's follow-up is advised. Nephrologists generally want to see their patients and check their renal function tests during the first half of Ramadan. However, holding clinics during holidays, such as the end of Ramadan and the Eid holiday, may pose logistical difficulties.

Study limitations. We did not have access to all nephrologists' contact information. In addition, our small sample size and low response rate limit generalization of the results. Self-administered questionnaire-based studies inherently lack flexibility and depth; comprehensive advice was not thus captured through our survey structure.

Further research is needed to explore nephrologists' perspectives on a larger scale, with more extensive interviews and qualitative analysis. Advanced CKD patients' ideas and concerns on fasting Ramadan even against medical advice should also be explored. The ultimate aim is to create guidelines that facilitate the care of fasting advanced CKD patients; taking into account both the current knowledge about the effect of fasting on CKD patients' medical condition, as well as nephrologists' and patients' perspectives.

In conclusion, the study addresses nephrologists' perspectives on fasting Ramadan for patients with advanced CKD. It highlights factors they consider when advising CKD patients on fasting, which were used to suggest applications in practice. Further studies are needed to comprehend nephrologists' and CKD patients' perspectives on fasting.

Furthermore we suggest: i) Holding a dedicated renal clinic before Ramadan is preferable to counsel advanced CKD patients seeking to fast. ii) The decision to fast should be individualized based on patient condition, risks and benefits of fasting. iii) The following patient factors have to be considered while advising advanced CKD patients regarding fasting Ramadan: eGFR, diabetes, age, use of diuretics, body fluid volume, and blood pressure. ix) Fasting CKD patients should be advised on appropriate hydration, rest, diet, complications, and medications adherence. v) Close monitoring of fasting CKD patients in terms of clinical and laboratory variables during and after Ramadan is highly recommended.

Acknowledgment. The authors would like to thank the Saudi Society of Nephrology and Transplantation (SSN) and the Saudi Center for Organ Transplantation (SCOT), Riyadh, Saudi Arabia for providing participants' contact information. We also appreciate the assistance of Mr. Mohammed Alkhateeb in data analysis. In addition, The authors extend their appreciation to the Deanship of Scientific Research at King Saud University for funding this work through the Undergraduate Student's Research Support Program, Project no. (URSP - 5 - 20 -01). We would also like to thank Scribendi (https://www.scribendi.com/) for English language editing. 


\section{References}

1. Ahmad S, Chowdhury TA. Fasting during Ramadan in people with chronic kidney disease: a review of the literature. Ther $A d v$ Endocrinol Metab 2019; 10: 2042018819889019.

2. Hill NR, Fatoba ST, Oke JL, Hirst JA, O'Callaghan CA, Lasserson DS, et al. Global prevalence of chronic kidney disease - A systematic review and meta-analysis. PLoS One 2016; 11: $\mathrm{e} 0158765$.

3. Pew-Templeton Global Religious Futures Project. The Future of World Religions: Population Growth Projections. [Updated 2016. Accessed 2020 August 1]. Available from URL: http:// www.globalreligiousfutures.org/

4. Bragazzi NL. Ramadan fasting and chronic kidney disease: does estimated glomerular filtration rate change after and before Ramadan? Insights from a mini meta-analysis. Int J Nephrol Renovasc Dis 2015; 8: 53-57.

5. Kara E, Sahin OZ, Kizilkaya B, Ozturk B, Pusuroglu G, Yildirim $S$, et al. Fasting in Ramadan is not associated with deterioration of chronic kidney disease: A prospective observational study. Saudi J Kidney Dis Transpl 2017; 28; 68-75.

6. Chowdhury A, Khan H, Lasker SS, Chowdhury TA. Fasting outcomes in people with diabetes and chronic kidney disease in East London during Ramadan 2018: the East London diabetes in Ramadan survey. Diabetes Res Clin Pract 2019; 152: 166-170.
7. Bakhit AA, Kurdi AM, Wadera JJ, Alsuwaida AO. Effects of Ramadan fasting on moderate to severe chronic kidney disease. A prospective observational study. Saudi Med J 2017; 38: 48-52.

8. Altamimi 1. Alzeghayer L, Tarakji AR. Factors affecting nephrologist's recommendation regarding fasting Ramadan for patients with advanced chronic kidney disease in Saudi Arabia (Sat-076). Kidney Int Rep 2020; 5: S1-S392.

9. Mbarki H, Tazi N, Najdi A, Tachfouti N, Arrayhani M, Sqalli T. Effects of fasting during Ramadan on renal function of patients with chronic kidney disease. Saudi J Kidney Dis Transpl 2015; 26: 320-324.

10. Hassan S, Hassan F, Abbas N, Hassan K, Khatib N, Edgim $\mathrm{R}$, et al. Does Ramadan fasting affect hydration status and kidney function in CKD patients? Ann Nutr Metab 2018; 72: 241-247.

11. Bernieh B, Al Hakim MR, Boobes Y, Abu Zidan FM. Fasting Ramadan in chronic kidney disease patients: clinical and biochemical effects. Saudi J Kidney Dis Transpl 2010; 21: 898-902.

12. International Diabetes Federation. Diabetes and Ramadan Practical guidelines. International Diabetes Federation (IDF), in collaboration with the Diabetes and Ramadan (DAR) International Alliance [Updated 2016 April. Accessed 2020 August 1]. Avaialable from URL: https://www. worlddiabetesfoundation.org/sites/default/files/IDF\%20 \%26\%20DAR\%20Guidelines\%20April-16-low_0.pdf 